\title{
Reasoning by SVD and Morphotronic Network
}

\author{
Zenon Chaczko and Germano Resconi
}

\begin{abstract}
The immune system of the vertebrates possess the capabilities of "intelligent" information processing, which include memory, the ability to learn, to recognize, and to make decisions with respect to unknown situations. The mathematical formalization of these capabilities forms the basis of immune-computing (IC) as a new computing approach that replicates the principles of information processing by proteins and immune networks. This IC approach looks rather constructive as a basis for a new kind of computing. With the Morphotronic System or the analogous SVD we can create effective learning process and create immune memory by the projection operators. Given the immune memory is possible to recognize and compare antigen in a way to take defense action to eliminate the dangerous cell.
\end{abstract}

\section{INTRODUCTION TO SVD}

W E assume reader's familiarity with the basic terminology of linear algebra, however, for more details related to the problem space refer to [1]-[6]. Our focus relates to application of matrices of real numbers as discussed by Tarakanov et al. [7]. The method can be used for management and optimization of resource utilization in the Wireless Sensor Network (Morphotronic network) [8], [9] that is composed of Intelligent Agents [1] adhering to minimum energy binding rules. If the superscript $T$ denotes the transpose of a vector or matrix, then two vectors $X$ and $Y$ are said to be orthogonal if the following condition is held true:

$$
X^{T} Y=0
$$

In $2 \mathrm{D}$ or $3 \mathrm{D}$ space, this simply means that the vectors are perpendicular. Now, let $A$ be a square matrix such that its columns are mutually orthogonal vectors of length 1 :

$$
X^{T} Y=1
$$

Then $A$ is an orthogonal matrix and

$$
A^{T} A=I
$$

$I$ is the identity matrix. To simplify the notation assume that a matrix $A$ has at least as many rows $M$ as columns $N$ where $M \geq N$. An SVD of an $M \times N$ matrix $A$ is any factorization of the following form:

$$
A=U \Sigma V^{T}
$$

where $U$ is an $M \times M$ orthogonal matrix $V$ is an $N \times N$ orthogonal matrix and $\Sigma$ is an $M \times N$ diagonal matrix with

$$
s_{i, j}=0 \quad \text { for } \quad i \neq j \quad \text { and } \quad s_{i, i}=s_{i} \geq 0
$$

Z. Chaczko is with the University of Technology, Sydney, Broadway NSW 2007, Australia (e-mail: zenon@eng.uts.edu.au).

G. Resconi is with Dept. of Mathematics and Physics, Catholic University Brescia, I-25121, Italy (e-mail: resconi@numerica.it).
Furthermore, it can be shown that there exist non unique matrices $U$ and $V$ such that

$$
s_{1} \geq s_{2} \geq \ldots \geq s_{N} \geq 0
$$

Henceforth, we will assume the SVD has such a property. The quantities $s_{i}$ are called the singular values of $A$ and the columns of $U$ and $V$ are called the left and right singular vectors respectively. For example, for the matrix:

$$
A=\left[\begin{array}{ll}
0.96 & 1.72 \\
2.28 & 0.96
\end{array}\right]
$$

the SVD can then be calculated as:

$$
A=U \Sigma V^{T}=\left[\begin{array}{cc}
0.6 & -0.8 \\
0.8 & 0.6
\end{array}\right]\left[\begin{array}{ll}
3 & 0 \\
0 & 1
\end{array}\right]\left[\begin{array}{cc}
0.8 & 0.6 \\
0.6 & -0.8
\end{array}\right]
$$

It can be demonstrated that the columns of $U$ and $V$ are unit length since

$$
(0.6)^{2}+(0.8)^{2}=1
$$

and a simple calculation of dot products will show them to be mutually orthogonal. From the components of the SVD many properties of the original matrix can be determined. The null space of a matrix $A$ is the set of $x$ for which $A x=0$ and the range of $A$ is the set of $b$ for which $A x=b$ has a solution for $x$.

Now, let $u_{j}$ and $v_{j}$ be the columns of $U$ and $V$ respectively, then the decomposition of:

$$
\begin{gathered}
A=U \Sigma V^{T} \quad \text { can be written as } \\
A v_{j}=s_{j} u_{j} \quad j=1,2, \ldots, N \\
\text { if } \quad s_{j}=0 \text { then } \quad A v_{j}=0
\end{gathered}
$$

and $v_{j}$ is in the null space of $A$, whereas $s_{j} \neq 0$.

\section{A. Properties of the SVD}

\section{1) SVD and Matrix Rank}

Fundamental to linear algebra is the notion of rank. Numerous theorems begin with this condition. If matrix $A$ is of full rank then the following property holds. However, if the matrix is rank deficient (or nearly so) then small perturbations of the matrix values from round off errors or fuzzy data will yield a matrix which is of full rank Hence, determining the rank of a matrix is non trivial. The SVD lends us a practical definition of rank as well as allows us to quantify the notion of near rank deficiency.

\section{2) SVD and Linear Independence}

Another use of the SVD technique provides a measure called a condition number which is related to the measure of 
linear independence between the column vectors of the matrix The condition number with respect to the Euclidean norm of a matrix $A$ is:

$$
\operatorname{cond}(A)=\frac{s_{\max }}{s_{\min }}
$$

where $s_{\min }$ and $s_{\max }$ are the largest and smallest singular values of $A$. Using the condition number we can quantify the independence of the columns of $A$.

\section{B. Applications of SVD}

\section{1) Solutions to Linear Equations}

Numerous practical problems can be expressed in the language of linear algebra. $A$ linear system involves a set of equations in $N$ variables [2]. For example, let's consider the following linear system defined as:

$$
\left\{\begin{array}{l}
x_{1}+2 x_{2}+x_{3}=8 \\
10 x_{1}+18 x_{2}+12 x_{3}=78 \\
20 x_{1}+22 x_{2}+40 x_{3}=144
\end{array}\right.
$$

This problem can be expressed in terms of a coefficient matrix $A$, where a vector $x$ of variables and a vector $b$ such that a solution to the linear system $A x=b$ is an assignment to the values of the vector $x$. For the above example $A x$ and bare:

$$
A x=\left[\begin{array}{ccc}
1 & 2 & 1 \\
10 & 18 & 12 \\
20 & 22 & 40
\end{array}\right]\left[\begin{array}{l}
x_{1} \\
x_{2} \\
x_{3}
\end{array}\right]=\left[\begin{array}{c}
8 \\
78 \\
144
\end{array}\right]=b
$$

Using the SVD of $A$ we can determine if a solution exists as well as the general form of the possible solutions $x$

$$
\text { If } U \Sigma V^{T} \text { is the } S V D \text { of } M x N \text { matrix } A(M \geq N)
$$

then, the system $A x=b$ becomes:

$$
U \Sigma V^{T} x=b
$$

Substituting $z=V^{T} x$ and $d=U^{T} b$ we have:

$$
\Sigma z=d
$$

Let $\operatorname{Rank}(A)=k$, where $k$ represents the number of non zero singular values $s_{i}$. Studying the linear equations of the diagonal system (1) we can determine whether or not there is a solution, which exists if and only if $d_{j}=0$ whenever $s_{j}=0$ or $j>N$. In cases where $k<N$, the $z_{j}$ associated with a zero $s, j$ can be set to any value and still yield a solution. A general form of the possible solutions can then be expressed in terms of these arbitrary components of $\mathrm{z}$ when transformed back to the original coordinates by $x=V z$. The condition number of a matrix can also describe the sensitivity of solutions of linear systems to inaccuracies in the data. An extension for this approach of solving linear systems can be a solution where $A x$ is approximately equal to $b$, by this we mean the least squares solution $x$ for optimization of results.

\section{2) Noisy Signal Filtering}

Problems in signal processing often use linear models for signals. In ideal (noise free) conditions the measurement data can be arranged in a matrix, where the matrix is known to be rank deficient. By this we mean that the signal is assumed to lie in a proper subspace of Euclidean space. However, the presence of noise either from rounding error or instrument error results in a measurement matrix that is often of full rank. Usually, the models assume that the error can be separated from the data in that the noise component is that which lies in a subspace orthogonal to the signal subspace. For this reason, the SVD is used to approximate the matrix decomposing the data into an optimal estimate of the signal and the noise components. Suppose $A$ is the measurement matrix, where each column consists of a signal component $\mathrm{x}$ and a noise component $n$ :

$$
A=\left(C_{1}\left|C_{2}\right| \ldots \mid C_{N}\right)
$$

where each $C_{i}=x_{i}+n_{i}$

The vector $x$ representing the signal, which is known to be located in a rank $k$ subspace, though the precise subspace is unknown. Therefore, let $x=H c$ for a coefficient vector $c$ and a matrix $H$ whose columns are the basis vectors of some rank $k$ subspace. The least squared error between $A$ and $H c$ is minimized by choosing $H$ to be the optimal $k$ rank approximation $A_{k}$ to $A$. Then the $k$ columns of $U$ corresponds to the $k$ largest singular values, span the rank $k$ subspace $H$. The resulting error can be denoted as:

$$
e^{2}=\sum_{k+1}^{N} s_{i}^{2}
$$

Using the SVD as above, we see that the original data matrix $A$ that can be decomposed into the orthogonal components

$$
U \Sigma_{n-k} V^{T}
$$

which corresponds to the orthogonal subspace defining the noise components.

\section{3) SVD and the Pseudoinverse}

The SVD analysis of a matrix $A$ gives an easy and uniform way of computing its inverse $A^{-1}$, whenever it exists, or its pseudoinverse $A^{+}$, otherwise. Therefore, if

$$
A=U \Sigma V^{T}
$$

then we have

$$
A^{+}=U \Sigma^{+} V^{T}
$$

The singular values $\sigma_{1}, \ldots, \sigma_{r}$ are locate on the diagonal of the matrix $\Sigma$, and the reciprocals of the singular values

$$
\frac{1}{\sigma_{1}}, \ldots, \frac{1}{\sigma_{r}}
$$

that are on the diagonal of the $n \times m$ matrix $\Sigma^{+}$. We know that for the pseudo inverse we have:

$$
A^{+} A^{+}=A
$$

For the square matrix and $A$ non singular

$$
\begin{gathered}
A^{+}=A^{-1} \\
A A^{+}=U U^{T}
\end{gathered}
$$


A

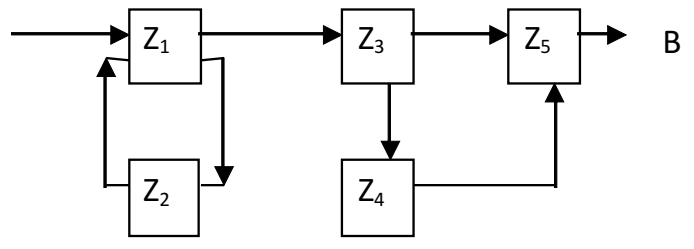

Fig. 1. Morphotronic network system.

$A A^{+} x=y$ where $y$ is the projection of $x$ into the subspace of the colons of $A$

$$
A A^{+} A=A, \quad A^{+} A A^{+}=A^{+}
$$

\section{Morphotronic Network System}

We define the Morphotronic structure as a network of input and output of $\mathrm{q} \times \mathrm{p}$ matrices where $q \geq p$. Refer to authors work in [2], [3].In Fig. 1 we demonstrate a single Morphotronic network.

$A=\left[\begin{array}{cccc}a_{11} & a_{12} & \ldots & a_{1 p} \\ a_{21} & a_{22} & \ldots & a_{2 p} \\ \ldots & \ldots & \ldots & \ldots \\ a_{q 1} & a_{q 2} & \ldots & a_{q p}\end{array}\right], B=\left[\begin{array}{cccc}b_{11} & b_{12} & \ldots & b_{1 p} \\ b_{21} & b_{22} & \ldots & b_{2 p} \\ \ldots & \ldots & \ldots & \ldots \\ b_{q 1} & b_{q 2} & \ldots & b_{q p}\end{array}\right]$

The graph representation of one module of the Morphotronic system is shown in Fig. 2.

Formally the input output between matrices in figure (2) is given by the expression

$$
B=Z A
$$

where

$$
Z=B\left(A^{T} A\right)^{-1} A^{T}
$$

In fact we have

$$
Z A=B\left(A^{T} A\right)^{-1} A^{T} A=B
$$

\section{A. Proposition 1:}

When $q>p$ for the same data $A$ and $B$ we have many different values of $Z$ for which

$$
B=Z A
$$

\section{B. Proof:}

For $Z=Z+L^{T}$ we have

$$
B=\left(Z+L^{T}\right) A=Z A+L A
$$

Now, when $L^{T} A=0$ with $L^{T}$ different from 0 we have that

$$
B=\left(Z+L^{T}\right) A=Z A+L^{T} A=Z A .
$$

thus, all the operators $Z+L^{T}$ transform $A$ into $B$. When we solve the homogeneous equation $L^{T} A=0$, or $\left(L^{T} A\right)^{T}=A^{T} L=0$ given $A$ we can compute the family
A

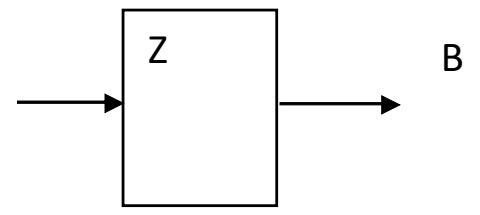

Fig. 2. Simple module in the Morphotronic network system.

of the transformations $Z+L^{T}$ that transform $A$ into $B$. For example given:

$$
A=\left[\begin{array}{ll}
1 & 0 \\
1 & 1 \\
0 & 1
\end{array}\right] \text { and } B=\left[\begin{array}{cc}
0 & 1 \\
1 & 1 \\
1 & 0
\end{array}\right]
$$

thus the solution of $A^{T} L=0$ is obtained as follows:

$$
A^{T} L=0
$$

when

$$
\begin{gathered}
{\left[\begin{array}{lll}
1 & 1 & 0 \\
0 & 1 & 1
\end{array}\right]\left[\begin{array}{l}
L_{1} \\
L_{2} \\
L_{3}
\end{array}\right]=0} \\
{\left[\begin{array}{ll}
1 & 1 \\
0 & 1
\end{array}\right]\left[\begin{array}{l}
L_{1} \\
L_{2}
\end{array}\right]+\left[\begin{array}{c}
0 \\
L_{3}
\end{array}\right]=0} \\
{\left[\begin{array}{ll}
1 & 1 \\
0 & 1
\end{array}\right]\left[\begin{array}{l}
L_{1} \\
L_{2}
\end{array}\right]=\left[\begin{array}{c}
0 \\
-L_{3}
\end{array}\right]}
\end{gathered}
$$

thus the solutions are:

$$
\begin{aligned}
& {\left[\begin{array}{l}
L_{1} \\
L_{2}
\end{array}\right]=\left[\begin{array}{ll}
1 & 1 \\
0 & 1
\end{array}\right]^{-1}\left[\begin{array}{c}
0 \\
-L_{3}
\end{array}\right]=} \\
& =\left[\begin{array}{cc}
1 & -1 \\
0 & 1
\end{array}\right]\left[\begin{array}{c}
0 \\
-L_{3}
\end{array}\right]=\left[\begin{array}{c}
L_{3} \\
-L_{3}
\end{array}\right]
\end{aligned}
$$

So

$$
\left[\begin{array}{l}
L_{1} \\
L_{2} \\
L_{3}
\end{array}\right]=\left[\begin{array}{c}
L_{3} \\
-L_{3} \\
L_{3}
\end{array}\right]
$$

Now, it can be concluded that:

$$
\begin{gathered}
A^{T} L=0, \quad L^{T} A=0 \quad \text { and } \\
B=\left(Z+L^{T}\right) A=Z A
\end{gathered}
$$

So, we have many different $Z$ that change $A$ into $B$.

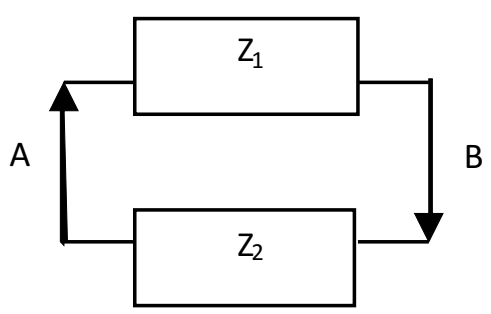

Fig. 3. Loop in the Morphotronic network system. 


\section{Loops and Projection Operators}

Now, let us study the Loop for the Morphotronic network (Fig. 3.).

For the equation (3) in the previous case we have

$$
\begin{gathered}
Z_{1}=B\left(A^{T} A\right)^{-1} A^{T} \\
Z_{2}=A\left(B^{T} B\right)^{-1} B^{T} \quad \text { and } \\
Q 1=Z_{1} Z_{2}=B\left(A^{T} A\right)^{-1} A^{T} A\left(B^{T} B\right)^{-1} B^{T}= \\
=B\left(B^{T} B\right)^{-1} B^{T}
\end{gathered}
$$

We have $Q 1 B=B$, and $Q 1$ is a projection operator with the following property:

$$
\begin{gathered}
Q 1^{2}=B\left(B^{T} B\right)^{-1} B^{T} B\left(B^{T} B\right)^{-1} B^{T}= \\
=B\left(B^{T} B\right)^{-1} B^{T}=Q 1
\end{gathered}
$$

In the same loop we have another projection operator Q2 defined in this way:

$$
\begin{gathered}
Q 2=Z_{2} Z_{1}=A\left(B^{T} B\right)^{-1} B^{T} B\left(A^{T} A\right)^{-1} A^{T}= \\
=A\left(A^{T} A\right)^{-1} A^{T}
\end{gathered}
$$

with $Q 2 A=A$, and

$$
\begin{gathered}
Q 2^{2}=A\left(A^{T} A\right)^{-1} A^{T} A\left(A^{T} A\right)^{-1} A^{T}= \\
A\left(A^{T} A\right)^{-1} A^{T}=Q 2
\end{gathered}
$$

\section{Geometric Image of the Projection Operator}

Now we show, in a simple case, the geometric image of the projection operator Q2 for the matrix.

$$
A=\left[\begin{array}{ll}
a_{1,1} & a_{1,2} \\
a_{2,1} & a_{2,2} \\
a_{3,1} & a_{3,2}
\end{array}\right]=A_{1} \oplus A_{2}=\left[\begin{array}{l}
a_{1,1} \\
a_{2,1} \\
a_{3,1}
\end{array}\right] \oplus\left[\begin{array}{l}
a_{1,2} \\
a_{2,2} \\
a_{3,2}
\end{array}\right]
$$

and the vector

$$
X=\left[\begin{array}{l}
X_{1} \\
X_{2} \\
X_{3}
\end{array}\right]
$$

we have

$$
\begin{gathered}
Q 2 X=A\left(A^{T} A\right) A^{T} X=A S= \\
=S_{1}\left[\begin{array}{c}
A_{1,1} \\
A_{2,1} \\
A_{3,1}
\end{array}\right]+S_{2}\left[\begin{array}{c}
A_{1,2} \\
A_{2,2} \\
A_{3,2}
\end{array}\right]=S_{1} A_{1}+S_{2} A_{2}
\end{gathered}
$$

and the geometric image of the projection operator is defined as depicted below (Fig. 4)

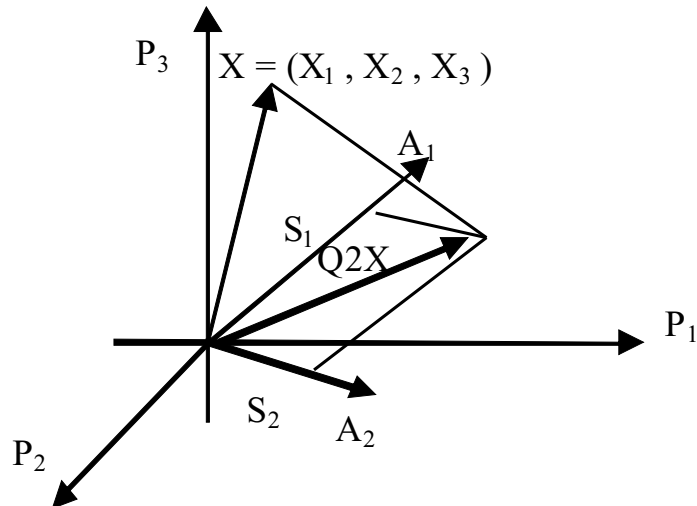

Fig. 4. Projection operator as loop in the Morphotronic system.

E. Another Projection Operator for the Same Loop

For $B=Z A$ we have

$$
Z=B\left(B^{T} A\right)^{-1} B^{T}
$$

In fact it can be denoted that:

$$
Z A=B\left(B^{T} A\right)^{-1} B^{T} A=B
$$

And in the loop in Fig. 3 for

$$
Z_{1}=B\left(B^{T} A\right)^{-1} B^{T} \quad \text { and } \quad Z_{2}=A\left(A^{T} B\right)^{-1} A^{T}
$$

We have the projection operator:

$$
\begin{gathered}
Q=Z_{2} Z_{1}=A\left(A^{T} B\right)^{-1} A^{T} B\left(B^{T} A\right)^{-1} B^{T}= \\
=A\left(B^{T} A\right)^{-1} B^{T}
\end{gathered}
$$

where

$$
Q A=A, \quad Q^{2}=A\left(B^{T} A\right)^{-1} B^{T} A\left(B^{T} A\right)^{-1} B^{T}=
$$

$$
=A\left(B^{T} A\right)^{-1} B^{T}=Q
$$

And we have another projection operator:

$$
\begin{gathered}
Q=Z_{1} Z_{2}=B\left(B^{T} A\right)^{-1} B^{T} A\left(A^{T} B\right)^{-1} A^{T}= \\
=B\left(A^{T} B\right)^{-1} A^{T}
\end{gathered}
$$

where

$$
\begin{gathered}
Q B=B, \quad Q^{2}=B\left(A^{T} B\right)^{-1} A^{T} B\left(A^{T} B\right)^{-1} A^{T}= \\
B\left(A^{T} B\right)^{-1} A^{T}=Q
\end{gathered}
$$

In conclusion for the same diagram in figure 3 we have four different type of projection operators. 


\section{SVD AS A SPECIAL CASE OF THE Morphotronic SYSTEM}

Given the matrix $A$, we can built the projection operator

$$
Q=A A^{+}=A\left(A^{T} A\right)^{-1} A^{T}
$$

Where $A^{+}$is the pseudo inverse of $A$. Now given the non singular quadratic matrix $M$, we have:

$$
\begin{gathered}
Q=(A M)(A M)^{+}=(A M)\left((A M)^{T}(A M)\right)^{-1}(A M)^{T}= \\
=A M M^{-1}\left(A^{T} A\right)^{-1}(M) A^{T}= \\
=A\left(A^{T} A\right)^{-1} A^{T}=A A^{+}
\end{gathered}
$$

For $M=V \Sigma^{-} 1$, where $V$ is the matrix of the eigenvectors of $A^{T} A$ and $\Sigma^{-1}$ is the a diagonal matrix of the inverse of the square of the eigenvalues of $A^{T} A$, and with well known properties of the eigenvectors and eigenvalues we have:

$$
\begin{aligned}
& (A M)^{T}(A M)=\left(M^{T}\right)\left(A^{T} A\right)(M)= \\
= & \left(V \Sigma^{-1}\right)^{T}\left(A^{T} A\right)\left(V \Sigma^{-1}\right)=\text { Identity }
\end{aligned}
$$

For example given

$$
\begin{gathered}
A=\left[\begin{array}{ll}
0.96 & 1.72 \\
2.28 & 0.96
\end{array}\right] \\
V=\left[\begin{array}{cc}
0.8 & 0.6 \\
0.6 & -0.8
\end{array}\right] \\
\Sigma^{-1}=\left[\begin{array}{ll}
\frac{1}{3} & 0 \\
0 & 1
\end{array}\right]
\end{gathered}
$$

we have

$$
M=V \Sigma^{-1}=\left[\begin{array}{cc}
0.8 & 0.6 \\
0.6 & -0.8
\end{array}\right] \Sigma^{-1}=\left[\begin{array}{cc}
\frac{1}{3} & 0 \\
0 & 1
\end{array}\right]
$$

and

$$
(A M)^{T}(A M)=\left[\begin{array}{ll}
1 & 0 \\
0 & 1
\end{array}\right]
$$

Now, we put $A M=U$, so for $M=V \Sigma^{-1}$ we have

$$
Q=\left(A V \Sigma^{-1}\right)\left(A V \Sigma^{-1}\right)^{+}=A V \Sigma^{-1}\left(A V \Sigma^{-1}\right)^{T}
$$

for

$$
A=U \Sigma V^{T}
$$

we have

$$
\begin{gathered}
Q=\left(U \Sigma V^{T} V \Sigma^{-1}\right)\left(U \Sigma V^{T} V \Sigma^{-1}\right)^{T}=U U^{T}= \\
=A A^{+}=A\left(A^{T} A\right)^{-1} A^{T}
\end{gathered}
$$

Now we have also that:

$$
Q=U U^{T}=(A M)(A M)^{T}
$$

for

$$
M=V \Sigma^{-1}
$$

we obtain

$$
Q=U U^{T}=\left(A V \Sigma^{-1}\right)\left(A V \Sigma^{1}\right)^{T}
$$

and

$$
U=A V \Sigma^{-1}
$$

$$
A=U \Sigma V^{T}
$$

It can be concluded that with the use of projection operator, the transformation $M$ can be found by the eigenvalues and eigenvectors of $A^{T} A$, and the SVD for $A$. A more general projection operator can be defined as:

$$
Q A=A, \quad Q=A\left(B^{T} A\right)^{-1} B^{T}
$$

where $A=R B$ we have:

$$
Q=R B\left(B^{T} R B\right)^{-1} B^{T}
$$

and for the transformation $U=B M$ we have

$$
\begin{gathered}
R(B M)\left((B M)^{T}(R B M)\right)^{-1}(B M)^{T}= \\
=R(B M)\left(\left(M^{T}\left(B^{T} R B\right) M\right)\right)^{-1}(B M)^{T}= \\
=R B M M^{-1}\left(B^{T} R B\right)^{-1}\left(M^{T}\right)^{-1}\left(M^{T}\right) B^{T}= \\
=R B\left(B^{T} R B\right)^{-1} B^{T}=Q
\end{gathered}
$$

with the change of $b$ into $B M$ the projection operator $Q$ is invariant. Now, we have to select $M$ in such a way that

$$
(B M)^{T} R B M=M^{T}\left(B^{T} R B\right) M=\text { Identity }
$$

Given the eigenvectors matrix $V$ of the matrix $B^{T} R B$ and the diagonal matrix of the square of the eigenvalues $\Sigma$ we set $M=V \Sigma^{-1}$ so for the properties of the eigenvalues and eigenvectors the following condition is true:

$$
M^{T}\left(B^{T} R B\right) M=I
$$

Now, the properties of $M$ can be revealed. In fact, with a mathematical computation we have:

$$
\begin{gathered}
B^{T} R B=\left(M^{T}\right)^{-1}\left(M^{T}\right)\left(B^{T} R B\right)(M)\left(M^{-1}\right)= \\
\left.=\left(M^{T}\right)^{-1}\left[M^{T} B^{T} R B\right) M\right]\left(M^{-1}\right)=\left(M^{T}\right)^{-1}\left(M^{-1}\right)
\end{gathered}
$$

so

$$
\left(M M^{T}\right)^{-1}=B^{T} R B
$$

and

$$
M M^{T}=\left(B^{T} R B\right)^{-1}
$$

Hence, we have the important property:

$$
M(B M)^{T}=M M^{T} B^{T}=\left(B^{T} R B\right)^{-1} B^{T}=B^{+}
$$

When $B M=U$, we obtain the traditional decomposition or SVD of the pseudo inverse of $B$ as follows:

$$
\begin{gathered}
V \Sigma^{-1} U^{T}=M(B M)^{T}=M M^{T} B^{T}= \\
=\left(B^{T} R B\right)^{-1} B^{T}=B^{+}
\end{gathered}
$$

Concluding, we are able to obtain these results also due to the fact that:

$$
B^{+} B=V \Sigma^{-1} U^{T}=\text { Identity }
$$

Hence we obtain:

$$
B=U \Sigma V^{T}
$$

That $i d$ the SVD of $B$ obtained by the morphotronic system definition of the projection operator and we also have:

$$
Q=R(B M)\left((B M)^{T}(R B M)\right)^{-1}(B M)^{T}=
$$




$$
\begin{gathered}
=R(B M)\left(\left(M^{T}\left(B^{T} R B\right) M\right)\right)^{-1}\left(B M^{T}\right)= \\
=R(B M)\left(B M^{T}\right)=R U U^{T}
\end{gathered}
$$

Sumarising, by $V, \Sigma, U$ we can define the pseudoinverse of $B$ in the projection operator and the projection operator itself. At the reverse by the eigenvalue and eigenvectors of $B^{T} R B$, we can obtain the $V, \Sigma, U$ in the SVD. It can be demonstrated that it is possible to define the SVD as a special case of the Morphotronic transformation loop or a projection operator.

\section{IMMUNO-COMPUTING MORPHOTRONICS AND SVD}

\section{A. Definition 1 (Principal).}

Cell is a pair $V=(f, P)$, where $f$ is a real value $f \in R$, whereas

$$
P=\left(p_{1}, p_{2}, \ldots, p_{q}\right)
$$

and where $P$ is a point of $q$-dimensional space: $P \in R^{q}$, and $P$ lies within unit cube:

$$
\begin{gathered}
\max \left\{\left|p_{1}\right|,\left|p_{2}\right|, \ldots,\left|p_{q}\right|\right\} \leq 1 \\
\max \{|p 1|, \ldots,|p q|\}
\end{gathered}
$$

\section{B. Definition 2}

The affinity is the distance $d_{i, j}\left(V_{i}, V_{j}\right)$ between cells

$$
V_{i}, V_{j}
$$

\section{Definition 3}

The cell $V_{i}$ recognizes cell $V_{j}$ if the following conditions are satisfied

$$
\begin{gathered}
\left|f_{i}-f_{j}\right|<\theta_{1} \\
d_{i, j}\left(V_{i}, V_{j}\right)<\theta_{2}
\end{gathered}
$$

where

$\theta_{1} \geq 0, \theta_{2} \geq 0$ are the recognition threshold and the affinity threshold.

\section{Definition 4}

The formal immune network (FIN) is a set of cells $W \subset W_{0}$ where $W_{0}$ is a set of cells that form the "innate immunity" controlled by the cytokine.

Rule 1 (apoptosis, elimination of redundancy)

If cell $V_{i}$ recognizes cell $V_{j}$ then remove $V_{i}$ for the set $W$

Rule 2 (autoimmunization or memory)

If cell $V_{k}$ is nearest to the cell $V_{i}$ among all cells in $W$

$$
d_{i, k}<d_{i, j}
$$

Whereas $\left|f_{i}-f_{k}\right| \geq \theta$ we have that $V_{i}$ is the most affine to $V_{i}$ but is beyond the recognition threshold. In this case in the Immuno-Computing (IC) we add $V_{i}$ to $W$.

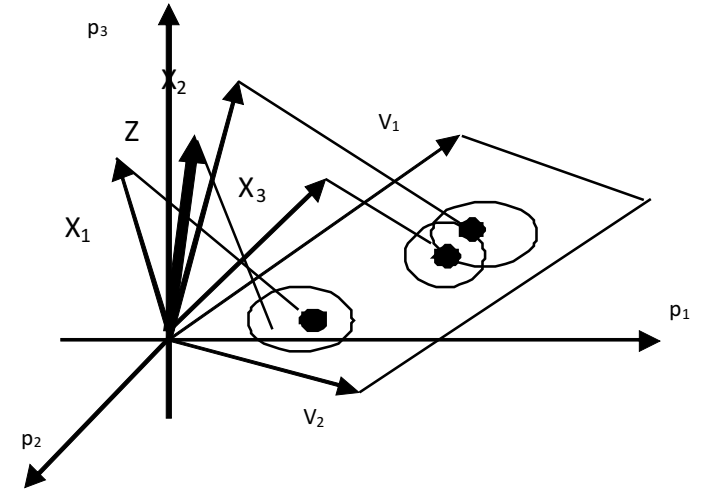

Fig. 5. Example of pattern recognition of $\mathrm{Z}$ in IC by Morphotronic loop or projection operator Q.

\section{E. Definition 5}

The "epitope" is defined as any point $P$ of the of the $q$ dimensional space of the cells. In this space we have the immune cells $W$ but we have also all the possible cells included the "antigen".

\section{PATtern ReCOGNition By IC-SVD AND Morphotronic Network System}

Given the matrix of the immune cells (FIN or formal immune network) in $W$ is expressed as:

$$
A=\left[\begin{array}{llll}
V_{1} & V_{2} & \ldots & V_{n}
\end{array}\right]=\left[\begin{array}{cccc}
v_{11} & v_{12} & \ldots & v_{1 n} \\
v_{21} & v_{22} & \ldots & v_{2 n} \\
\ldots & \ldots & \ldots & \ldots \\
v_{q 1} & v_{q 2} & \ldots & v_{q n}
\end{array}\right]
$$

and that the matrix of the Training Vectors (antigenic training) is denoted as:

$$
X=\left[\begin{array}{llll}
X_{1} & X_{2} & \ldots & X_{m}
\end{array}\right]=\left[\begin{array}{cccc}
x_{11} & x_{12} & \ldots & x_{1 m} \\
x_{21} & x_{22} & \ldots & x_{2 m} \\
\ldots & \ldots & \ldots & \ldots \\
x_{q 1} & x_{q 2} & \ldots & x_{q m}
\end{array}\right]
$$

The training vectors can be projected into the space of the immune cells (FIN) by the projection operator expressed as:

$$
\begin{gathered}
Y=Q X=A\left(A^{T} A\right)^{-1} A^{T} X \\
\text { by } S V D A=U \Sigma V^{T} \text { we were } \\
Y=Q X=U U^{T} X
\end{gathered}
$$

Given the training matrix $X$ of samples, projected in the space of the immune cells (or FIN) in order to obtain the learning process for the immune system, in three dimension of the $q$ space, the points of the projections, can be depicted as a $Z$ pattern recognition by IC using the projection operator $Q$ (Fig. 5).

When the learning cycle is completed a mature immune system is obtained. Given a new cell $Z$ we can project this cell into the immune system and verify where is the training projection or the immune cell that is affined to $Z$. The immune cell that is affined with the projection of $Z$ activate all the immune system to destroy the antigen $Z$. 
IC/SVD-based Minimum Energy Binding Clustering Algorithm for Sensor Network Clustering

- Obtain test set to determine goal of which to form cluster $(\mathrm{X}, \mathrm{Y}, \mathrm{Z})$ dimensions of sink - i.e. 3 dimensions by default)

- Obtain training set to determine cluster formation (X,Y,Z dimensions - i.e. 3 dimensions by default)

- Perform SVD transformation on training set and return 3 largest singular values

- For each value 'v' in the right singular value matrix o Perform a dot vector operation on the inverted test set, right singular value of ' $v$ ' divided by the Eigen-values in the matrix of dimension ' $v, v$ ' and return the results as binding energies vector

- Perform a Euclidean distance weight funcion of the inverted binding energies and the left singular value matrix and return as a single-dimension Euclidean distance weighting

- Sort the Euclidean distance weighting in ascending order, such that the node ID with the lowest binding energy is ranked highestas closest bound to the test set

Fig. 6. The main application of the Minimum Energy Binding Clustering algorithm is to initiate and organise the process of IC-SVD reasoning in autonomic clustering of the sensor network (Morphotronic system).

\section{OPTIMIZATION OF WSN CLUSTERING USING IC/SVD METHOD IN MORPHOTRONIC NETWORK}

One of many potential applications of the presented ICSVD and Morphotronic network technique is resource (energy) management in Wirelesses Sensor Networks (WSN).

Special case of the Morphotronic transformation and given sets $A$ of premises and set $B$ in the conclusion of the fuzzy rules, we can make Validation tests of several variants of IC/SVD based algorithms (Fig.6) for sensor clustering in sensor networks indicate resource (energy) efficiencies when evaluated as an expression of the number of transactions per of SVD-based clustering. These efficiencies are significantly greater when compared to traditional non-cluster head optimization methods [4]. Similarly, according to the results drawn for simulated throughput, the IC/SVD based routing protocols can also demonstrate significant improvements in sensornet energy management if benefits of the minimum binding energy approach are extended to data routing algorithms.

The study of resource (energy) efficiencies can be assisted by visual representations of energy (resource) distribution and usage in the wireless sensor network field (Fig. 7 and Fig. 8) that are generated by SVD-based Minimum Energy Binding (MEB) algorithms [4]. The main application of the Minimum Energy Binding Clustering algorithm is to initiate and organise the process of IC-SVD reasoning in autonomic sensor clustering and routing in the Morphotronic sensornet.

\section{CONCLUSION}

In this paper we deliver a comprehensive background for the SVD decomposition of a matrix A and we demonstrate some of its important application. Furthermore, it is argued that the concept of IC defined by A. Tarakanov et al [7] is

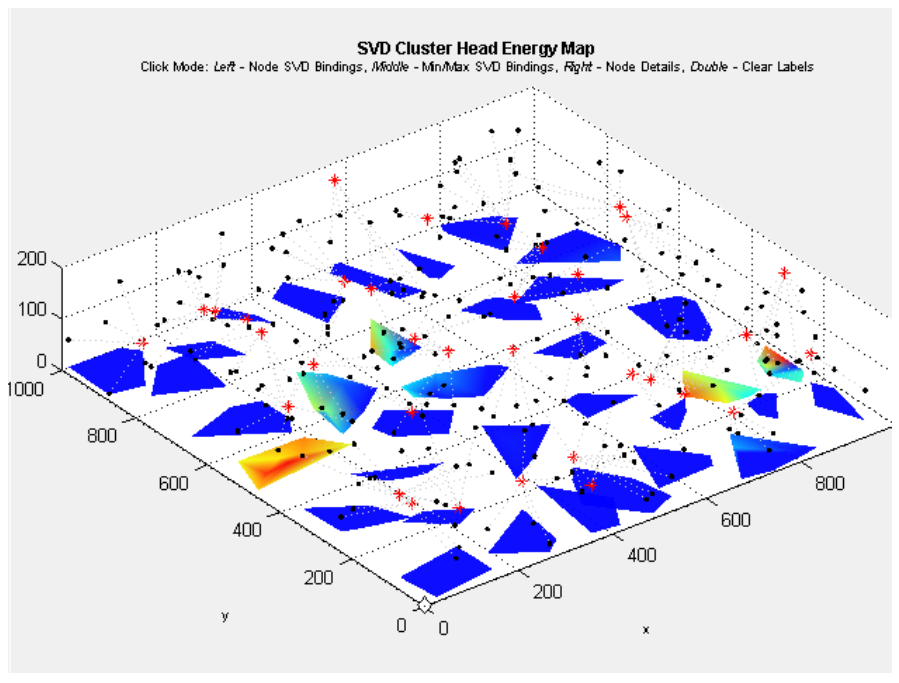

Fig. 7. Minimal Energy Binding patterns for efficient clustering of sensors in WSNs [4].

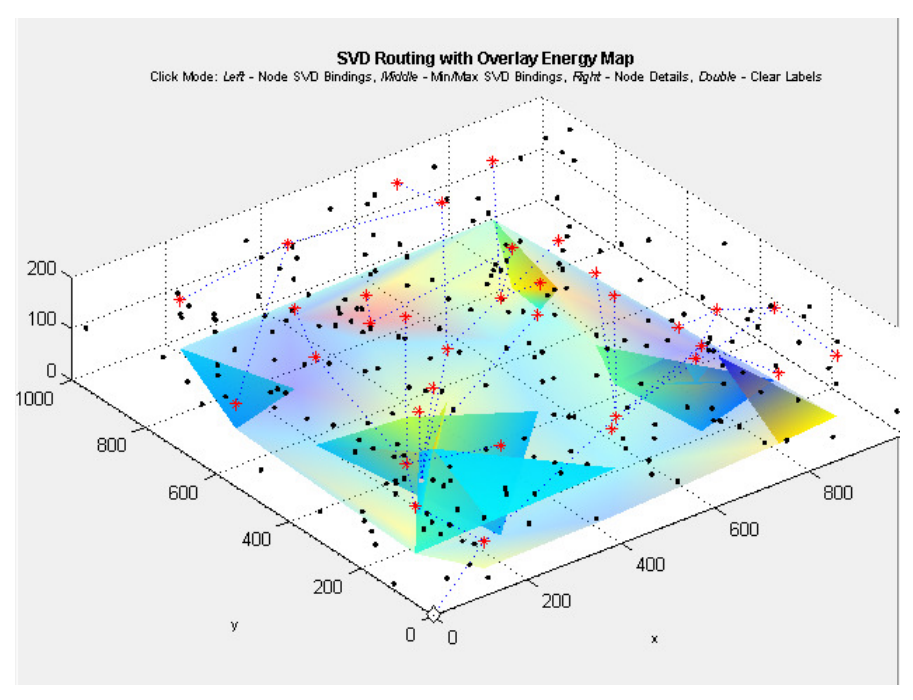

Fig. 8. Minimal Energy Binding patterns for energy efficient data routing in WSNs [4].

a special application of a more general concept denoted as the Morphotronic system theory that includes all the properties of the SVD. SVD based tools can be used to simplify the computation in the Morphotronic network such as WSN. Experiments conducted with IC/SVD based algorithms for collaborative clustering and routing in sensornets indicate potential benefits of the technique for autonomic optimization of resource (energy) utilization and improved network management [4].

\section{REFERENCES}

[1] F. Deprettere (Eds), SVD and signal processing: algorithms, applications and architectures. Institute of Electrical and Electronics Engineers: European Association for Signal Processing, 1988.

[2] G. Resconi and Z. Chaczko, "Morphotronic Systems Theory," in Proceedings of Computer Aided Systems Theory, Eurocast'09, LNCS, Las Palmas, Spain, 2009.

[3] L. M. Surhone, M. T. Timpledon, and S. F. Marseken, Singular Value Decomposition: Linear Algebra, Matrix Decomposition, Signal Processing, Statistics, Moore-Penrose Pseudoinverse, Least Squares, Diagonal Matrix, Unitary Matrix, Spectral Theorem. BetaScript Publishers, 2009. 
[4] Z. Chaczko, "Towards epistemic autonomy in adaptive biomimetic middleware for cooperative sensornets," Ph.D. dissertation, University of Technology, Sydney (UTS), Australia, July 2009.

[5] Z. Chaczko and G. Resconi, "Morphotronic Systems Applications," in Proceedings of Computer Aided Systems Theory, Eurocast'09, LNCS, Las Palmas, Spain, 2009.

[6] - "Organising Software Infrastructures: EgoMorphic BIM Model," International Journal of Computing Anticipatory Systems, Chaos, vol. 21, pp. 372-385, 2008, publ. by Chaos.

[7] A. O. Tarakanov, V. A. Skormin, and S. S. Sokolova, Immunocomputing: Principles \& Applications. Springer Verlag, 2003.
[8] G. Resconi and A. J. van der Wal, Morphogenetic Neural Network, ser Series Studies in Fuzziness and Soft Computing. Springer-Verlag, July 2007, ch. M. Nikravesh, L. A. Zadeh and J. Kacprzyk, Forging the new Frontieres: Fuzzy Pioneers I.

[9] G. Resconi, "The Morphogenetic Systems in Risk Analysis," in Proceeding of the International Conference on Risk Analysis and Crisis Response, Shangchai, China, 25-26 September 2007, pp. 161-165. 